\title{
Persistent Pain After Operative Treatment for Chronic Lateral Ankle Instability
}

\author{
Byung-Hyun Ahn' \\ Byung-Ki Cho $\mathbb{D}^{1,2}$
}

'Department of Orthopaedic Surgery, Chungbuk National University Hospital, Cheongju, South Korea; ${ }^{2}$ Department of Orthopaedic Surgery, College of Medicine, Chungbuk National University, Cheongju, South Korea
Correspondence: Byung-Ki Cho Department of Orthopaedic Surgery, Chungbuk National University Hospital,

62 Gaesin-dong, Seowon-gu, Cheongju,

Chungbuk, 28644, South Korea

Tel +82-43-269-6077

Fax +82-43-274-87I 9

Email titanick25@naver.com

\begin{abstract}
A high frequency of associated injuries is seen in patients with chronic lateral ankle instability. Comorbidities include intraarticular pathologies (osteochondral lesion, soft tissue or bony impingement syndrome, loose body, synovitis, etc.), peroneal tendon pathologies, neural injuries, and other extraarticular pathologies. Surgeons should have a high index of suspicion for these associated pathologies before operative intervention, correlate with clinical findings, and plan the treatment. Despite the restoration of ankle stability following ligament repair or reconstruction surgery, postoperative residual pain, which can negatively affect clinical outcomes and patient satisfaction, is highly prevalent (13-35\%). The aim of this review was to discuss the causes of persistent pain after operative treatment for chronic lateral ankle instability.
\end{abstract}

Keywords: ankle, chronic lateral instability, operative treatment, persistent pain

\section{Introduction}

Lateral ankle sprain is the most common musculoskeletal injury in athletic populations, accounting for up to $30 \%$ of sports injuries. ${ }^{1}$ Appropriate initial treatment and education are important to prevent recurrent episodes of acute ankle sprain, but a significant proportion of patients develop re-injury, progressing to chronic ankle instability. ${ }^{2-5}$ The most common symptoms of patients with chronic ankle instability are usually repetitive episodes of "giving way" or functional instability, which is expressed as a feeling of disability or anxiety in the ankle joint. ${ }^{2,6}$ They commonly complain of difficulties with walking on uneven ground and athletic activities. There is a spectrum of symptoms in chronic ankle instability, of which pain is not a predominant symptom but can be an underrated symptom. Although injuries to intraarticular or surrounding structures often accompany ankle sprain and can cause chronic pain or dysfunction, a careful approach in the initial stage is lacking in actual clinical practice. In a recent systematic review, most studies reported that ankle pain was present in $50 \%$ to $79 \%$ of participants with chronic ankle instability. ${ }^{7}$ Those pain was usually mild and intermittent and related to strenuous physical activity. ${ }^{7}$ Surgical treatment is applied after comprehensive consideration of the degree of mechanical instability of the ankle joint evaluated by a surgeon and results of rehabilitation. Patients with ankle instability and chronic pain during walking or activity must be carefully screened for peroneal tendon injury, deltoid ligament or distal tibiofibular syndesmosis injury, osteochondral lesions, and traction injury of the nerves around the ankle joint, which are often accompanied by repetitive lateral ankle sprain (Figure 1). ${ }^{6-12}$ Careful history-taking and physical examination are the most important diagnostic methods not to miss accompanying pathologies. Various pathologies should be considered as causes of chronic ankle pain. 


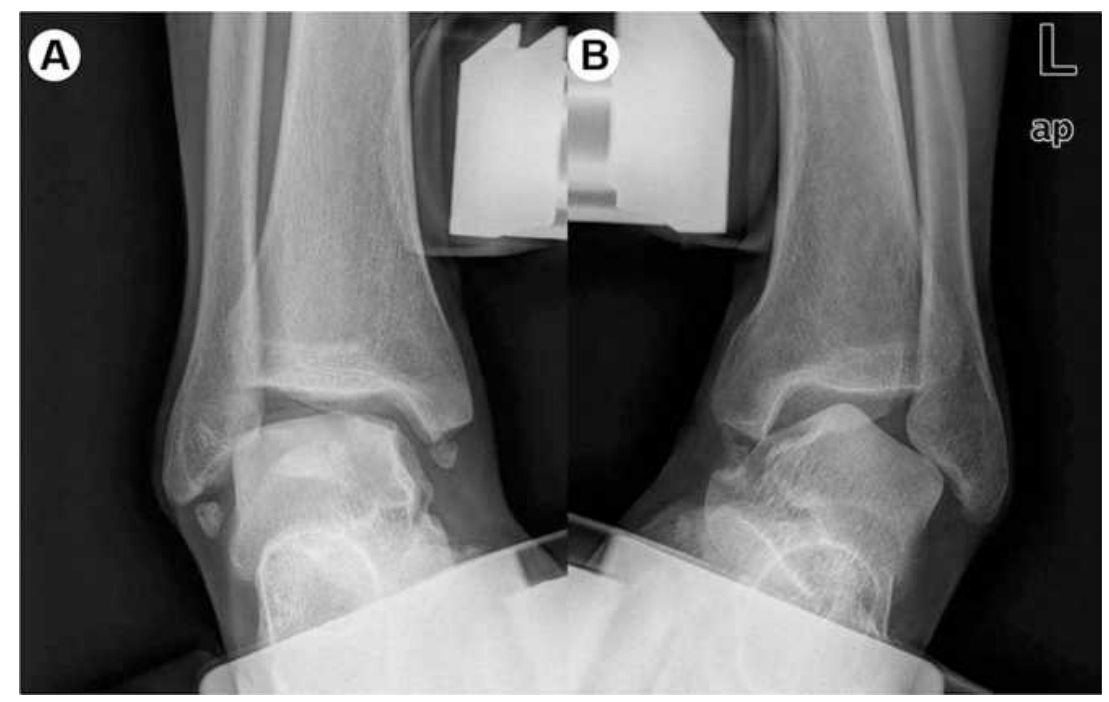

Figure I (A and B) Accompanying pathologies secondary to repetitive lateral ankle sprain.

The most representative surgical method to date for chronic ankle instability has been the modified Broström procedure, which restores mechanical stability through anatomical repair of the lateral ligaments of the ankle joint. The modified Broström procedure has relatively fewer complications and several advantages, with satisfactory clinical outcomes of approximately $90-95 \% .^{2,13}$ However, many patients (13-35\%) complain of persistent pain despite good recovery of joint stability with operative treatment for chronic ankle instability. ${ }^{12,14-17}$ In addition to restoring ankle joint stability, pain relief in daily living and sports activities are major factors enabling a successful surgical treatment, and proper additional procedures are often required with ligament surgery. In this article, we describe the causes of residual pain after operative treatment for chronic lateral ankle instability in three major categories: insufficient diagnosis, ineffective procedure, and iatrogenic complications.

\section{Unrecognized Pathologies at the Time of Surgery}

Before surgical treatment for chronic ankle instability, the accurate diagnosis should be made based on the presence of intraarticular pathologies, such as cartilage or osteochondral lesions (Figure 2), synovitis, and loose bodies. It is also necessary to determine whether or not the patient's symptom is associated with these lesions. Furthermore, detailed examination and diagnosis of instability caused by deltoid ligament or syndesmosis injury and symptoms associated with nerve or tendon injury around the ankle joint are required. It is usually necessary to check the degree of rupture of the lateral ligaments and condition of the remnant ligamentous tissue, cartilage, and surrounding soft tissue on magnetic resonance imaging (MRI) preoperatively.

\section{Intraarticular Pathologies}

Intraarticular pathologies (Box 1) accompanied by chronic ankle instability occur at a high frequency of approximately $63-95 \%$ in arthroscopic examinations, ${ }^{9,11,12,15,18-20}$ and failure to make an accurate diagnosis can lead to chronic discomfort and degenerative changes in the ankle joint. ${ }^{11,12,17,21-23}$ Therefore, careful preoperative examination of intraarticular lesions and appropriate treatment are critical to obtain satisfactory clinical results after surgery for ankle instability. According to a study by Ferkel et al, only 20\% of intraarticular lesions are accessible through capsulotomy during the modified Broström operation. ${ }^{15}$ Hintermann et al reported that more than $50 \%$ of patients with chronic ankle instability had

Box I Common Intra-Articular Lesions Associated with Chronic Lateral Ankle Instability

Osteochondral lesions of the talus (OLT): cartilage fibrillation, cartilage or osteochondral defect

Osteochondral lesions of the tibial plafond

Loose bodies

Anterolateral soft tissue impingement: hypertrophic synovial tissue interposition

Anterior tibial plafond or talar osteophyte (anterior bony

impingement)

Chronic synovitis

Distal tibiofibular syndesmosis instability 


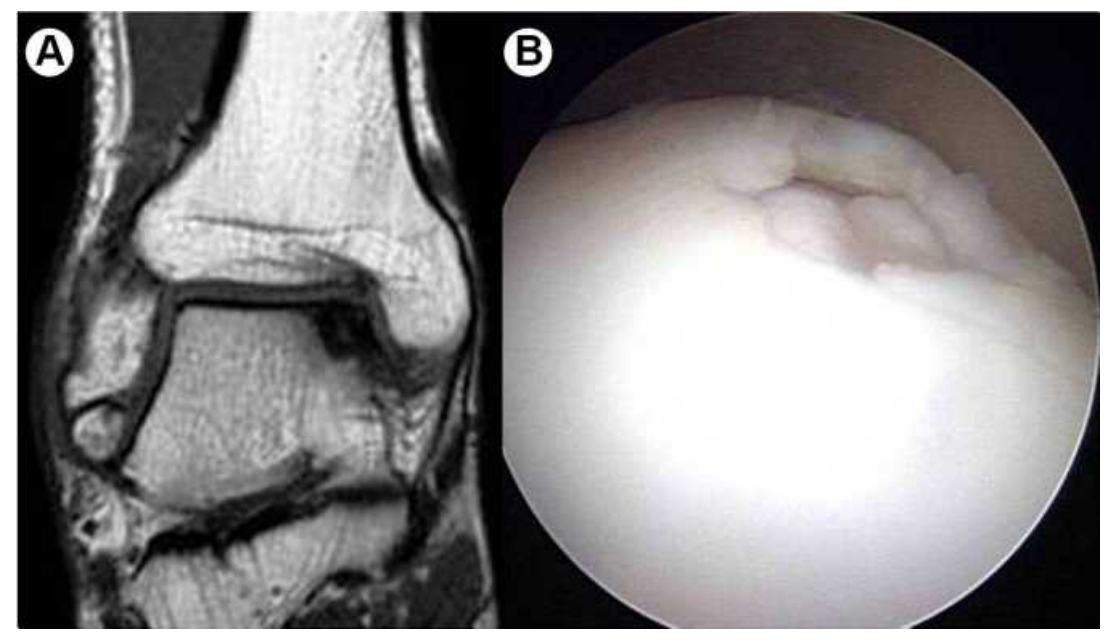

Figure 2 ( $\mathbf{A}$ and $\mathbf{B}$ ) Osteochondral lesion of the talar dome accompanied by chronic ankle instability.

osteochondral lesions of the talus in arthroscopic examination, but only $4 \%$ of the patients could be diagnosed based on preoperative physical examination and radiographs. ${ }^{9}$ In addition, chondral lesions not found preoperatively were observed at a considerable frequency in the tibial plafond $(8 \%)$, medial malleolus (11\%), and lateral malleolus (2.5\%). Considering the high prevalence of intraarticular lesions accompanying chronic ankle instability and the possibility of a preoperative missed diagnosis, arthroscopic procedures during ligament repair or reconstruction surgery can be an essential complement. ${ }^{9,10,15,24}$

The frequency of osteochondral lesions accompanied by chronic ankle instability is approximately $17-85 \%,{ }^{9-11,17,25}$ and they affect the medial joint surface rather than the lateral aspect of the tibial plafond or talus. In many cases, it may be hard to detect them in simple radiographs (Figure 3). In particular, osteochondral lesions in the tibial plafond are easily overlooked. $^{26}$ Okuda et al reported that the degree of ankle instability, age, and sex were not related to the presence of accompanying osteochondral lesions. ${ }^{11}$ Wang et al reported a higher risk of osteochondral lesions or osteophyte formation in association with older age, a post-injury period of more than 5 years, and male sex. ${ }^{25}$ In patients with chronic ankle instability, the frequency of synovitis with associated fibrous scar tissue is $32-100 \%$ in arthroscopic examination $^{8,14,18,27}$ and usually occurs in the anterior

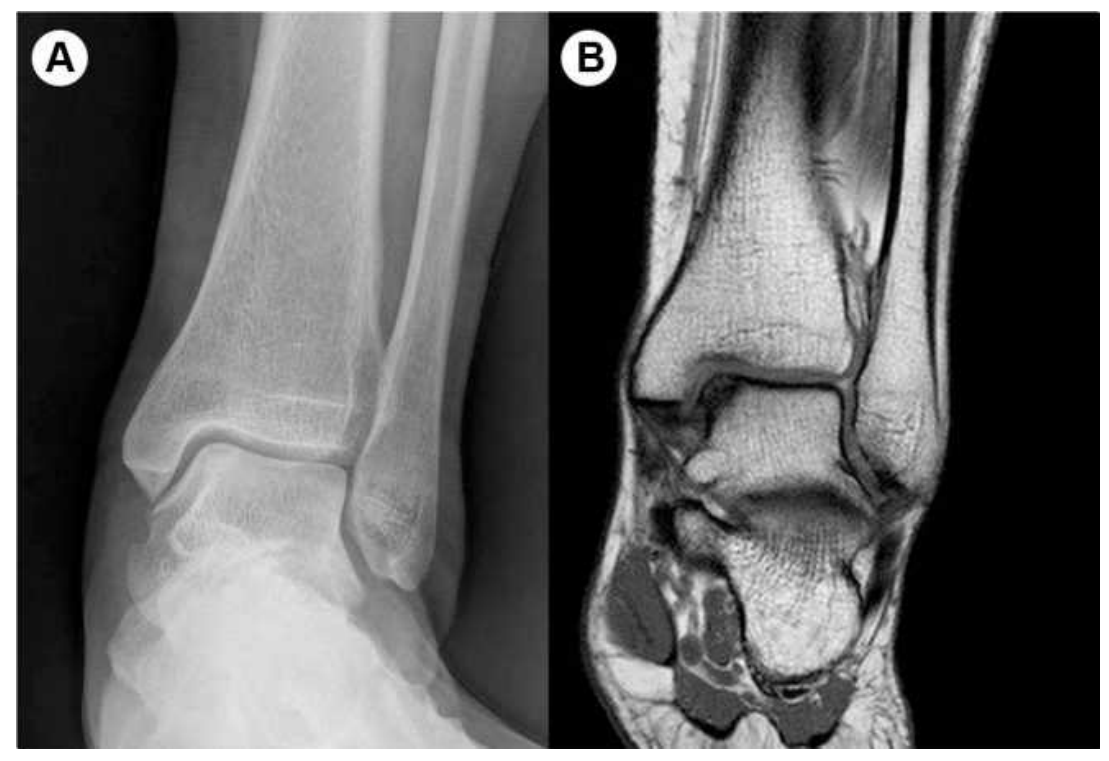

Figure 3 (A and B) Osteochondral lesion difficult to be diagnosed in preoperative radiographic examination. 
and anterolateral aspects of the ankle joint (Figure 4). Bony impingement syndrome caused by osteophyte formation at the anterior tibial plafond and talar neck occurs in approximately $11-19 \%$ of patients with chronic ankle instability (Figures 5 and 6). ${ }^{10,15,18,23,25,27}$ This lesion is considered to be an adaptive response to restore joint stability against increased anterior translation of the talus in chronic ankle instability. Moon et al reported that osteophyte size was closely related to osteochondral lesion severity and that approximately $81 \%$ of patients with osteophytes had an accompanying osteochondral lesion. ${ }^{28}$ It is also relatively common to overlook distal tibiofibular syndesmosis injury. Lateral ankle sprain accompanies syndesmosis injury in approximately $10 \%$ of patients and more frequently in professional athletes. ${ }^{2,14}$ It is important to consider that syndesmosis instability causes repetitive ankle sprains and may result in chronic pain. ${ }^{29}$

MRI is useful to accurately evaluate osteochondral lesions among intraarticular pathologies accompanied by chronic ankle instability. The diagnostic value of MRI for soft tissue lesions, such as impingement syndrome and synovitis, remains controversial. ${ }^{30,31}$ In a study comparing preoperative MRI findings with surgical findings, Staats et al reported that MRI showed overall low sensitivity and high specificity in the diagnosis of accompanying lesions other than osteochondral lesions; therefore, MRI alone cannot replace arthroscopic examination. ${ }^{32}$ In a similarly designed study by O'Neill et al, preoperative MRI interpreted by radiologists showed only $39 \%$ of chondral lesions, $56 \%$ of peroneal tendon problems, and $57 \%$ of intraarticular loose bodies. ${ }^{24}$ The overall sensitivity was

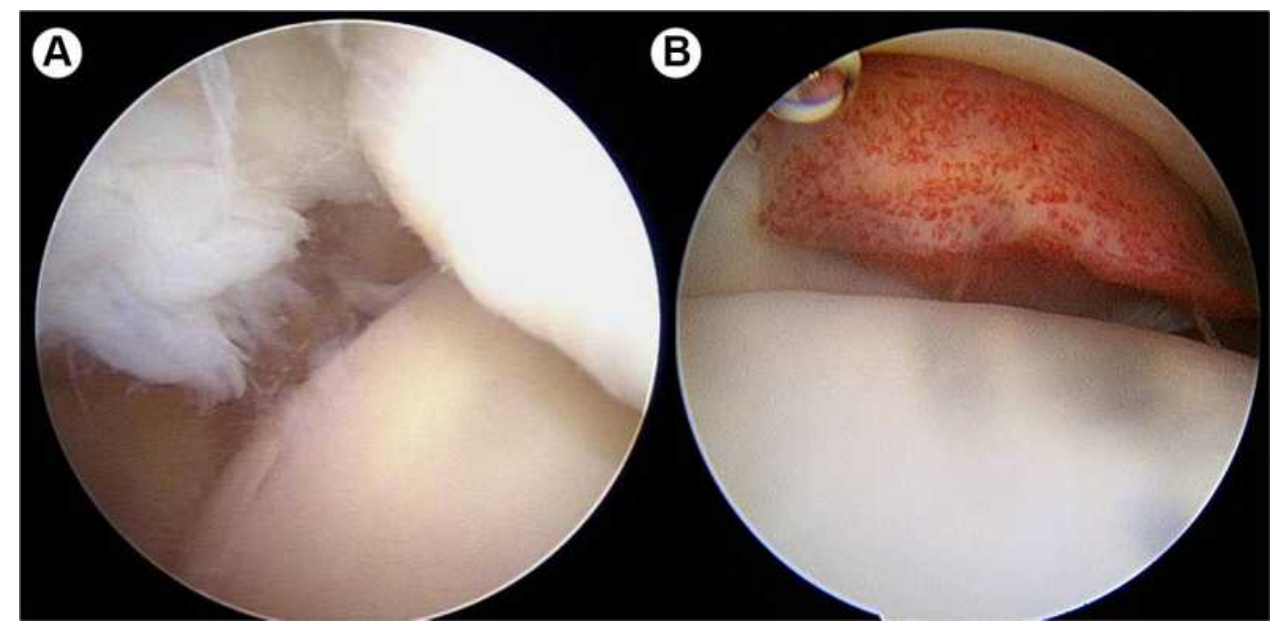

Figure 4 (A and B) Fibrous scar tissue and synovitis accompanied by chronic ankle instability.

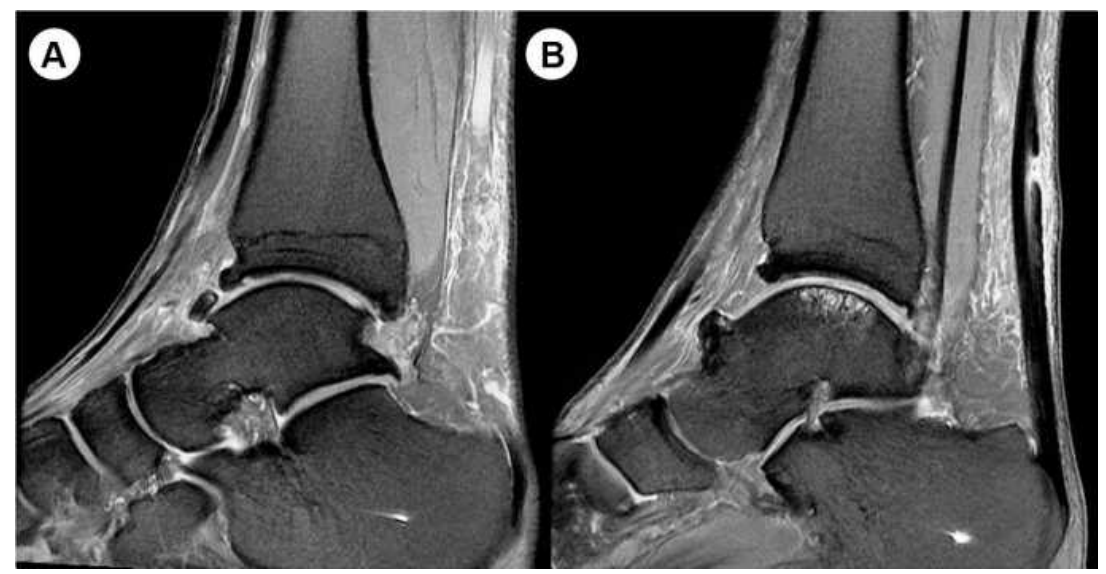

Figure 5 (A and B) Anterior bony impingement (spurs) accompanied by chronic ankle instability. 


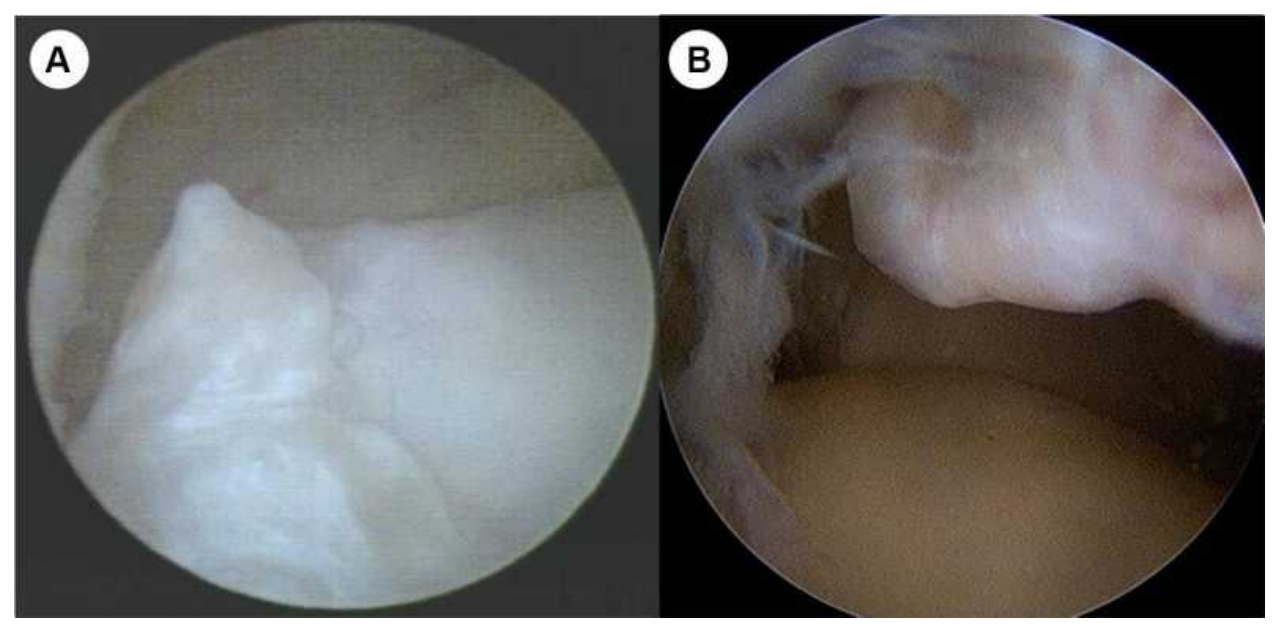

Figure 6 (A and B) Osteophytes formation at the anterior tibial plafond and the talar neck.

approximately $45 \%$. Orthopedic surgeons diagnosed $47 \%$ of chondral lesions, $71 \%$ of peroneal tendon problems, and $89 \%$ of intraarticular loose bodies on preoperative MRI. The overall sensitivity was approximately $63 \%$, not showing sufficient validity.

\section{Peroneal Tendon Problems}

Peroneal tendon problems concomitant with chronic ankle instability include peroneal tenosynovitis, peroneal tendon split tear, and attenuated peroneal retinaculum (Figure 7). DiGiovanni et al reported that approximately $77 \%$ of patients with chronic ankle instability had these peroneal tendon pathologies. ${ }^{8}$ Strauss et al reported that the most common extraarticular lesion accompanied by chronic ankle instability was the peroneal tendon problem (28\%), and untreated peroneal tendon lesions were the most common cause of postoperative residual pain. ${ }^{33}$ Based on this high incidence, some authors have recommended visually inspecting peroneal tendons in all patients during surgery for chronic ankle instability. Odak et al reported that approximately $5 \%$ of patients with chronic ankle instability showed tibialis posterior tenosynovitis on preoperative MRI, which should be considered when evaluating medial ankle pain. ${ }^{18}$

\section{Neuropathy Around the Ankle Joint}

In cases of ankle sprains, stretching injury of the superficial peroneal nerve by excessive inversion and plantar

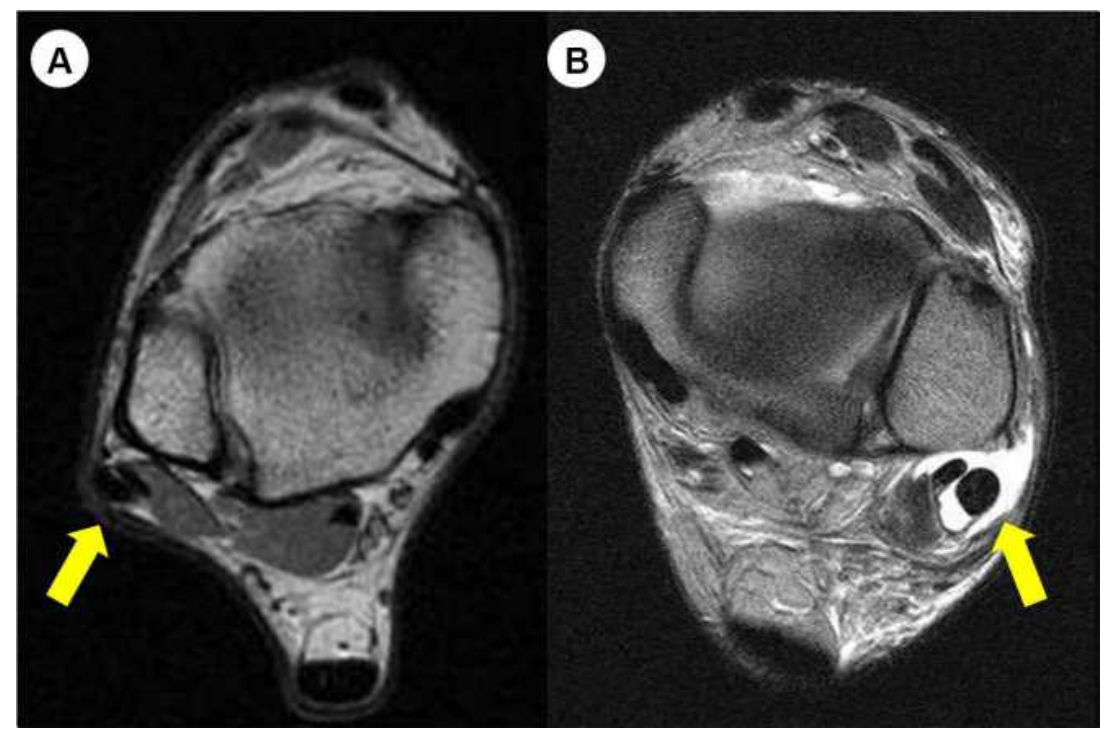

Figure 7 (A and $\mathbf{B})$ Peroneal tendon subluxation and tenosynovitis in patients with chronic ankle instability. 
flexion of the ankle is relatively common. ${ }^{34}$ According to a study by Nitz et al, electromyography following acute ankle sprain showed peroneal nerve injury in $86 \%$ of patients with severe ankle sprain while tibial nerve injury in $83 \%{ }^{35}$ If chronic symptoms persist because of nerve injury caused by acute ankle sprain, diagnostic methods include Tinel's test, electromyography and nerve conduction velocity, and the nerve block test (lidocaine block test). Careful history-taking and a high index of suspicion for the possibility of nerve damage are important to avoid a misdiagnosis.

\section{Other Extraarticular Pathologies}

It is important to keep in mind whether or not a patient has extraarticular pathologies, such as cavovarus deformity of the hindfoot (Figure 8), generalized ligamentous laxity, joint hypermobility, weakness in the ankle dorsiflexor or evertor, Achilles tendon tightness, and tarsal coalition. If these extraarticular lesions are not recognized, possibilities of recurrence of instability and residual pain postoperatively may be high. ${ }^{8,33}$ Strauss et al reported that the most commonly associated condition among extraarticular lesions contributing either to continued pain or recurrent instability was hindfoot varus alignment abnormalities followed by peroneal injuries. ${ }^{33}$ In addition,

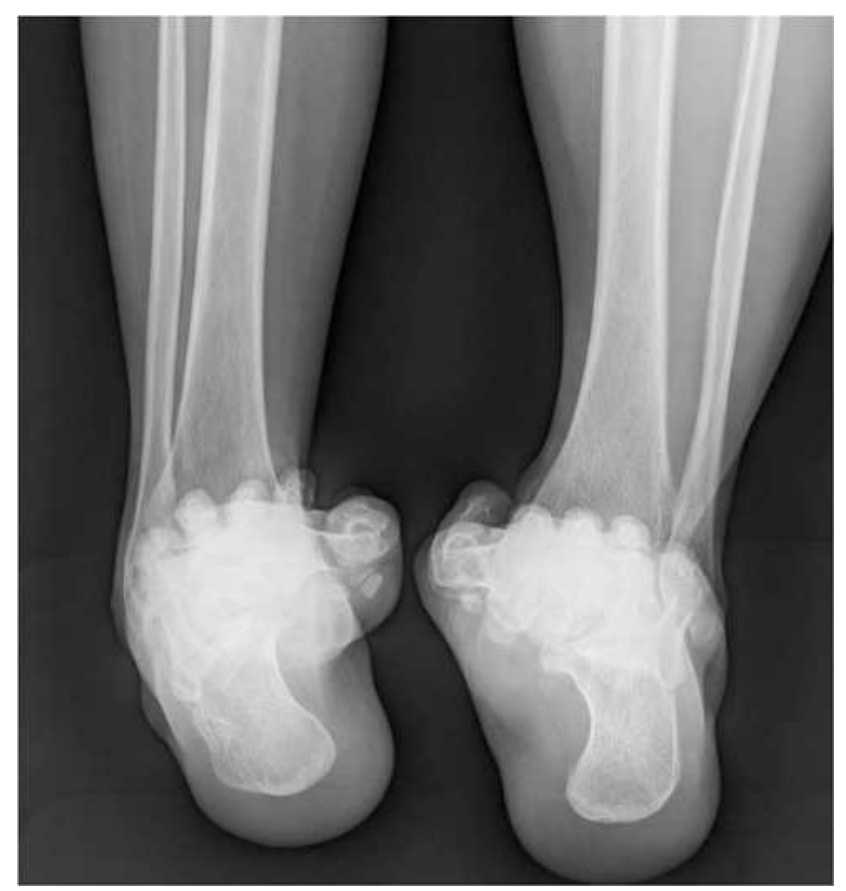

Figure 8 Varus malalignment of the hindfoot accompanied by chronic ankle instability. they identified other extraarticular conditions contributing to pain associated with lateral ankle instability: os trigonum and anterior tibial spurs. Patients with tarsal coalition often complain of recurrent ankle sprain with flatfoot deformity and midfoot pain (Figure 9). ${ }^{36}$ Subtalar and lateral ankle instabilities manifest similar symptoms, and pain in the sinus tarsi area can occur after repeated ankle sprains. $^{37}$

\section{Unresolved Pathologies with Surgical Treatments}

Clinical outcomes evaluation after the surgical treatment for chronic ankle instability usually includes restoration of joint stability, improvement of pain, better functional ability in daily and sport activities. Because there is few research specifically addressing pain among the clinical outcomes after the surgical treatment for chronic ankle instability, the interpretation of postoperative clinical outcomes in previous literatures may be restrictive to comprehend the changes of pain. Clinical results after the surgical treatment for chronic ankle instability and arthroscopic procedure for intra-articular lesions are relatively satisfactory. ${ }^{11,14,15,20,23,33}$ However, recurrence of the instability occurs at a frequency of approximately $5-10 \%$ after lateral ankle ligament repair or reconstruction. ${ }^{2,13}$ In addition, clinical results of arthroscopic procedures (synovectomy, chondroplasty, microfracture, multiple drilling, spur excision, and loose body removal) may vary depending on the operator's expertise and severity of lesions. According to a study by Choi et al, syndesmosis instability, osteochondral lesions of the talus, and subfibular ossicles were prognostic factors negatively affecting postoperative clinical outcomes. ${ }^{14}$ In contrast, the presence of soft tissue impingement or osteophytes which showed the highest incidence (81.5\%) among accompanying lesions, had little effect on clinical outcomes. ${ }^{14}$ Komenda and Ferkel reported that increasing the number of accompanying intraarticular lesions negatively affected the postoperative prognosis and that treatment for an osteochondral lesion of the talus was the most important factor for postoperative clinical outcomes. ${ }^{10}$ Hua et al reported significantly lower clinical evaluation scores (American Orthopaedic Foot and Ankle Society score) in patients with osteochondral lesions along with chronic ankle instability and concluded that the presence of an osteochondral lesion was the most important prognostic factor. ${ }^{20}$ In contrast, Okuda et al reported that the presence 


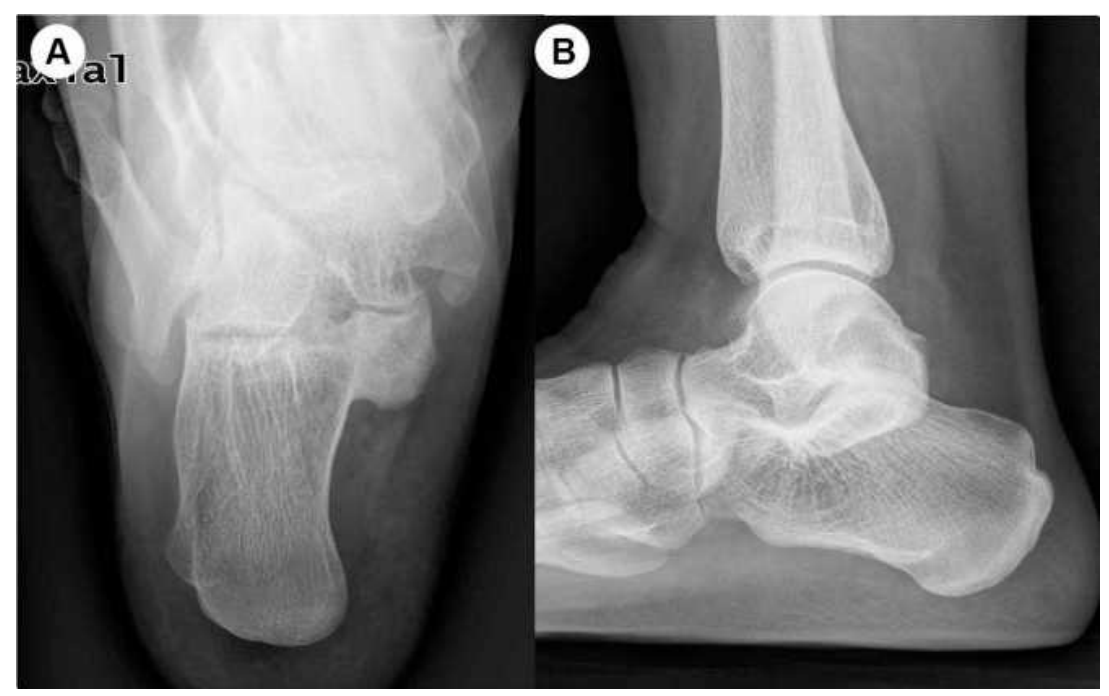

Figure 9 (A and B) Talocalcaneal coalition in a patient with chronic ankle instability.

of a focal osteochondral lesion had little effect on midterm clinical and radiologic outcomes after ligament reconstruction for chronic ankle instability. ${ }^{11}$ Although the duration of ankle instability and the severity of intraarticular lesions (particularly, osteochondral lesion of the talus) are not clearly correlated, most authors agree that prolonged ankle instability can increase the risk of repeated sprain injuries and number of intraarticular lesions. $^{25}$ Lee et al reported that male patients, patients with body mass index (BMI) greater than $30 \mathrm{~kg} / \mathrm{m}^{2}$, and older patients have a high probability of having two or more accompanying intraarticular lesions. ${ }^{27}$ Scranton et al reported that removal of osteophytes (anterior body impingement) in the anterior tibial plafond or talar neck had little effect on clinical outcomes, ${ }^{38}$ while Cannon et al reported better clinical results after removal of osteophytes. $^{39}$

If surgical treatment is performed in middle-aged patients with long-standing lateral ankle instability, ankle pain during walking and exercise may persist because of degenerative changes already in progress, even if joint stability is well restored postoperatively. ${ }^{34,40,41}$ Takao et al conducted anatomical ligament reconstruction with an autologous gracilis tendon graft and arthroscopic multiple drilling in patients with degenerative arthritis accompanied by chronic ankle instability and found excellent clinical results (87.4 points on the AOFAS scale) in stage 2 of the classification by Takakura. However, they concluded that these combined procedures could not be recommended for patients with moderate and severe degenerative arthritis, based on unsatisfactory clinical results (61.2 points on the
AOFAS scale) in stage $3 .^{42}$ Cho et al performed the modified Broström procedure and arthroscopic debridement in middle-aged patients with Takakura stage 2, showing significantly improved clinical outcomes. Nevertheless, osteoarthritis progressed in approximately $27.3 \%$ of patients during the mid-term follow-up of average 56.2 months, and residual pain at walking persisted in $36.4 \%$ of patients. ${ }^{40}$ In the aforementioned study by Cho et al, the recurrence rate of postoperative ankle instability was as relatively low as $4.5 \%$. Therefore, aggravation of the arthritis grade was analyzed to mainly result from degenerative changes in progress rather than recurrence of ankle instability. It is necessary to clearly distinguish degenerative changes from osteochondral lesion accompanying chronic ankle instability to select the appropriate surgical treatment, and a more realistic approach is required for patients with degenerative arthritis above the moderate degree. ${ }^{41}$ In addition, if only simple abrasion chondroplasty or microfracture is performed in osteochondral lesions with a full-thickness chondral defect or subchondral cyst, and large osteochondral lesions, this may be inadequate to successfully regenerate cartilage and lead to degenerative arthritis (Figure 10). Progression to degenerative arthritis is found in approximately $33 \%$ of patients at the long-term follow-up after arthroscopic bone marrow stimulation for osteochondral lesions of the talus. ${ }^{43,44}$ Considering the characteristics (size, depth, location, subchondral cyst, etc.) of osteochondral lesions and patient age, it is important to apply appropriate surgical treatments, such as osteochondral autologous transplantation, autologous chondrocyte implantation and chondrogenesis, to reduce residual pain postoperatively. 


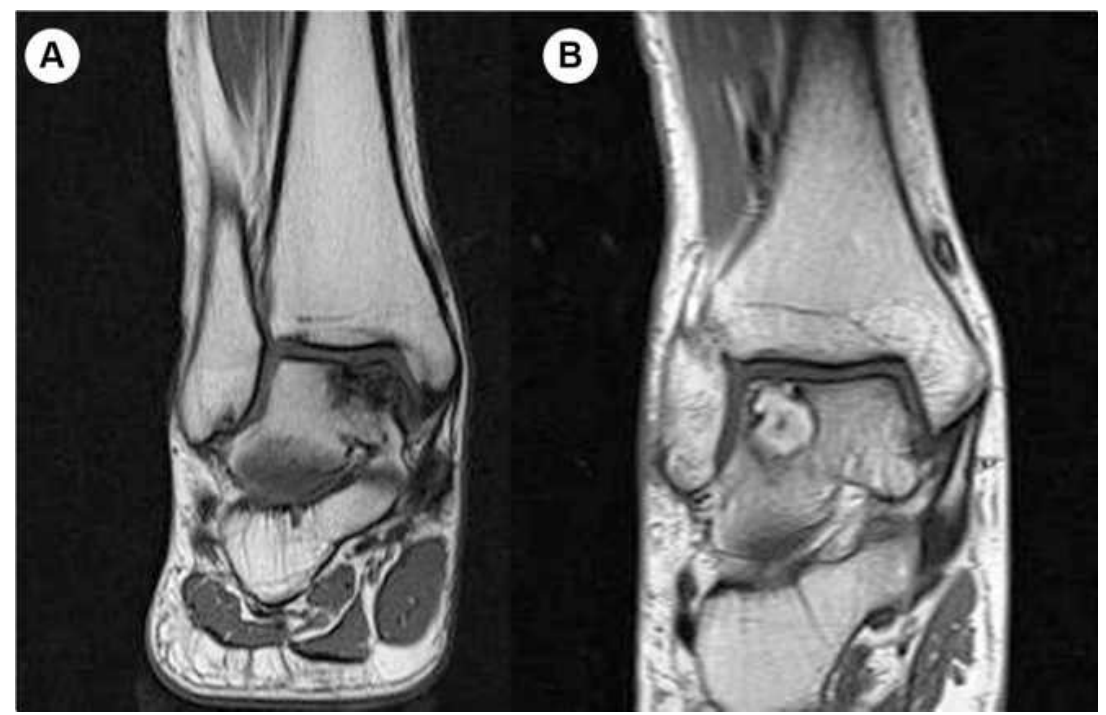

Figure 10 (A and B) Large-sized osteochondral lesion and subchondral cyst difficult to obtain successful regeneration of cartilage with only bone-marrow stimulation techniques.

\section{latrogenic Pathologies by Surgical Procedures}

Ligament surgery or arthroscopic treatment for chronic ankle instability can result in a new kind of pain that the patient did not experience preoperatively, and this can become a troublesome factor affecting the clinical result and patient satisfaction. Vega et al reported the intraoperative iatrogenic cartilage damage rate to be $31 \%$ of their cases, and $6.7 \%$ of them invaded more than $50 \%$ of cartilage depth. ${ }^{45}$ In the aforementioned study, the most frequently damaged areas were central and anterolateral aspects of the talar dome, and $65 \%$ of the total injuries occurred during intraarticular procedures and other $35 \%$ occurred during portal creation for arthroscope insertion. Intraoperative peripheral nerve damage around the ankle joint has been commonly reported with an occurrence rate of $7-19 \%$ and is caused by skin incision and soft tissue dissection during ligament repair or reconstruction surgeries, damage adjacent to the arthroscope insertion portal, and excessive traction applied during operation. ${ }^{46}$ The most commonly injured nerve is the lateral branch of the superficial peroneal nerve. Sural and saphenous nerve damages have also been reported. ${ }^{34}$ Although uncommonly reported, atypical neurologic symptoms or severe pain out of proportion to the patient's condition should raise suspicion for diagnosis of complex regional pain syndrome (CRPS). A recent systematic review by Guelfi et al showed that the complication rate following the modified Broström operation was 7.9\%, which mainly comprised local wound problems, sensory nerve dysfunction, and prolonged pain. ${ }^{13}$ The complication rate related to the arthroscopic Broström operation was $15.3 \%$, which mainly comprised superficial peroneal nerve damage, delayed wound healing, residual instability, and skin irritation by non-absorbable suture materials. ${ }^{13}$

\section{Conclusion}

The restoration of joint stability and pain relief in daily and sport activities are the main factors influencing the clinical outcomes and patient satisfaction after the surgical treatment for chronic lateral ankle instability, which is the reason why concomitant treatments, such as arthroscopic procedures along with ligament surgery, are required. Various intraarticular lesions and adjacent tendon and nerve injuries, which commonly accompany chronic lateral ankle instability, should be evaluated with careful history-taking, physical examination, radiographs, and MRI. Persistent pain postoperatively should raise suspicion for various pathologies that require differential diagnosis, and a clear decision on correlation with patient's symptoms is warranted.

\section{Funding}

The authors declare that they have no funding in this work.

\section{Disclosure}

The authors report no conflicts of interest related to this work. 


\section{References}

1. Fong DT, Hong Y, Chan LK, Yung PS, Chan KM. A systematic review on ankle injury and ankle sprain in sports. Sports Med. 2007;37(1):73-94. doi:10.2165/00007256-200737010-00006

2. DiGiovanni CW, Brodsky A. Current concepts: lateral ankle instability. Foot Ankle Int. 2006;27(10):854-866. doi:10.1177/ 107110070602701019

3. Hubbard TJ. Ligament laxity following inversion injury with and without chronic ankle instability. Foot Ankle Int. 2008;29 (3):305-311. doi:10.3113/FAI.2008.0305

4. O'Loughlin PF, Murawski CD, Egan C, Kennedy JG. Ankle instability in sports. Phys Sportsmed. 2009;37(2):93-103. doi:10.3810/ psm.2009.06.1715

5. Gribble PA, Bleakley CM, Caulfield BM, et al. Evidence review for the 2016 international ankle consortium consensus statement on the prevalence, impact and long-term consequences of lateral ankle sprains. Br J Sports Med. 2016;50(24):1496-1505. doi:10.1136/ bjsports-2016-096189

6. Michels F, Pereira H, Calder J, et al. Searching for consensus in the approach to patients with chronic lateral ankle instability: ask the expert. Knee Surg Sports Traumatol Arthrosc. 2018;26 (7):2095-2102. doi:10.1007/s00167-017-4556-0

7. Al Adal S, Pourkazemi F, Mackey M, Hiller CE. The prevalence of pain in people with chronic ankle instability: a systematic review. $J$ Athl Train. 2019;54(6):662-670. doi:10.4085/10626050-531-17

8. DIGiovanni BF, Fraga CJ, Cohen BE, Shereff MJ. Associated injuries found in chronic lateral ankle instability. Foot Ankle Int. 2000;21 (10):809-815. doi:10.1177/107110070002101003

9. Hintermann B, Boss A, Schafer D. Arthroscopic findings in patients with chronic ankle instability. Am J Sports Med. 2002;30(3):402-409. doi: $10.1177 / 03635465020300031601$

10. Komenda GA, Ferkel RD. Arthroscopic findings associated with the unstable ankle. Foot Ankle Int. 1999;20(11):708-713. doi:10.1177/ 107110079902001106

11. Okuda R, Kinoshita M, Morikawa J, Yasuda T, Abe M Arthroscopic findings in chronic lateral ankle instability: do focal chondral lesions influence the results of ligament reconstruction? Am J Sports Med. 2005;33(1):35-42. doi:10.1177/036354 6504271058

12. Taga I, Shino K, Inoue M, Nakata K, Maeda A. Articular cartilage lesions in ankles with lateral ligament injury: an arthroscopic study. Am J Sports Med. 1993;21(1):120-126. doi:10.1177/03635465 9302100120

13. Guelfi M, Zamperetti M, Pantalone A, Usuelli FG, Salini V, Oliva XM. Open and arthroscopic lateral ligament repair for treatment of chronic ankle instability: a systematic review. Foot Ankle Surg. 2018;24(1):11-18. doi:10.1016/j.fas.2016.05.315

14. Choi WJ, Lee JW, Han SH, Kim BS, Lee SK. Chronic lateral ankle instability: the effect of intra-articular lesions on clinical outcome. Am J Sports Med. 2008;36(11):2167-2172. doi:10.1177/ 0363546508319050

15. Ferkel RD, Chams RN. Chronic lateral instability: arthroscopic findings and long-term results. Foot Ankle Int. 2007;28(1):24-31. doi:10.3113/FAI.2007.0005

16. Gregush RV, Ferkel RD. Treatment of the unstable ankle with an osteochondral lesion: results and long-term follow-up. Am J Sports Med. 2010;38(4):782-790. doi:10.1177/0363546509351556

17. Sugimoto K, Takakura Y, Okahashi K, Samoto N, Kawate K, Iwai M. Chondral injuries of the ankle with recurrent lateral instability: an arthroscopic study. J Bone Joint Surg Am. 2009;91(1):99-106. doi:10.2106/JBJS.G.00087
18. Odak S, Ahluwalia R, Shivarathre DG, et al. Arthroscopic evaluation of impingement and osteochondral lesions in chronic lateral ankle instability. Foot Ankle Int. 2015;36(9):1045-1049. doi:10.1177/ 1071100715585525

19. Takao M, Uchio Y, Naito K, Fukazawa I, Ochi M. Arthroscopic assessment for intra-articular disorders in residual ankle disability after sprain. Am J Sports Med. 2005;33(5):686-692. doi:10.1177/ 0363546504270566

20. Hua Y, Chen S, Li Y, Chen J, Li H. Combination of modified Broström procedure with ankle arthroscopy for chronic ankle instability accompanied by intra-articular symptoms. Arthroscopy. 2010;26(4):524-528. doi:10.1016/j.arthro.2010.02.002

21. Wikstrom EA, Hubbard-Turner T, McKeon PO. Understanding and treating lateral ankle sprains and their consequences. Sports Med. 2013;43(6):385-393. doi:10.1007/s40279-013-0043-z

22. Valderrabano V, Hintermann B, Horisberger M, Fung TS. Ligamentous posttraumatic ankle osteoarthritis. Am J Sports Med. 2006;34(4):612-620. doi:10.1177/0363546505281813

23. Nery C, Raduan F, Del Buono A, Asaumi ID, Cohen M, Maffulli N. Arthroscopic-assisted brostrom-gould for chronic ankle instability: a long-term follow-up. Am J Sports Med. 2011;39(11):2381-2388. doi: $10.1177 / 0363546511416069$

24. O'Neill PJ, van Aman SE, Guyton GP. Is MRI adequate to detect lesions in patients with ankle instability? Clin Orthop Relat Res. 2010;468(4):1115-1119. doi:10.1007/s11999-009-1131-0

25. Wang DY, Jiao C, Ao YF, et al. Risk factors for osteochondral lesions and osteophytes in chronic lateral ankle instability: a case series of 1169 patients. Orthop J Sports Med. 2020;8(5):2325967120922821. doi:10.1177/2325967120922821

26. Mologne TS, Ferkel RD. Arthroscopic treatment of osteochondral lesions of the distal tibia. Foot Ankle Int. 2007;28(8):865-872. doi:10.3113/FAI.2007.0865

27. Lee J, Hamilton G, Ford L. Associated intra-articular ankle pathologies in patients with chronic lateral ankle instability: arthroscopic findings at the time of lateral ankle reconstruction. Foot Ankle Spec. 2011;4(5):284-289. doi:10.1177/1938640011416355

28. Moon JS, Lee K, Lee HS, Lee WC. Cartilage lesions in anterior bony impingement of the ankle. Arthroscopy. 2010;26(7):984-989. doi:10.1016/j.arthro.2009.11.021

29. Williams GN, Jones MH, Amendola A. Syndesmotic ankle sprains in athletes. Am J Sports Med. 2007;35(7):1197-1207. doi:10.1177/ 0363546507302545

30. Meislin RJ, Rose DJ, Parisien JS, Springer S. Arthroscopic treatment of synovial impingement of the ankle. Am J Sports Med. 1993;21 (2):186-189. doi:10.1177/036354659302100204

31. Lee JW, Suh JS, Huh YM, Moon ES, Kim SJ. Soft tissue impingement syndrome of the ankle: diagnostic efficacy of MRI and clinical results after arthroscopic treatment. Foot Ankle Int. 2004;25 (12):896-902. doi:10.1177/107110070402501209

32. Staats K, Sabeti-Aschraf M, Apprich S, et al. Preoperative MRI is helpful but not sufficient to detect associated lesions in patients with chronic ankle instability. Knee Surg Sports Traumatol Arthrosc. 2018;26(7):2103-2109. doi:10.1007/s00167-017-4567-x

33. Strauss JE, Forsberg JA, Lippert FG. Chronic lateral ankle instability and associated conditions: a rationale for treatment. Foot Ankle Int. 2007;28(10):1041-1044. doi:10.3113/FAI.2007.1041

34. O'Neill PJ, Parks BG, Walsh R, Simmons LM, Miller SD. Excursion and strain of the superficial peroneal nerve during inversion ankle sprain. J Bone Joint Surg Am. 2007;89(5):979-986. doi:10.2106/ 00004623-200705000-00009

35. Nitz AJ, Dobner JJ, Kersey D. Nerve injury and grades II and III ankle sprains. Am J Sports Med. 1985;13(3):177-182. doi:10.1177/ 036354658501300306 
36. Snyder RB, Lipscomb AB, Johnston RK. The relationship of tarsal coalitions to ankle sprains in athletes. Am J Sports Med. 1981;9 (5):313-317. doi:10.1177/036354658100900505

37. Aynardi M, Pedowitz DI, Raikin SM. Subtalar instability. Foot Ankle Clin. 2015;20(2):243-252. doi:10.1016/j.fcl.2015.02.007

38. Scranton PE Jr, McDermott JE, Rogers JV. The relationship between chronic ankle instability and variations in mortise anatomy and impingement spurs. Foot Ankle Int. 2000;21:657-664.

39. Cannon LB, Hackney RG. Anterior tibiotalar impingement associated with chronic ankle instability. J Foot Ankle Surg. 2000;39 (6):383-386. doi:10.1016/S1067-2516(00)80074-5

40. Cho BK, Shin YD, Park HW. Outcome following a modified Broström procedure and arthroscopic debridement of medial gutter osteoarthritis combined with chronic ankle instability. Foot Ankle Int. 2018;39(12):1473-1480. doi:10.1177/1071100718793395

41. Irwin TA, Anderson RB, Davis WH, Cohen BE. Effect of ankle arthritis on clinical outcome of lateral ankle ligament reconstruction in cavovarus feet. Foot Ankle Int. 2010;31(11):941-948. doi:10.3113/ FAI.2010.0941
42. Takao M, Komatsu F, Naito K, Uchio Y, Ochi M. Reconstruction of lateral ligament with arthroscopic drilling for treatment of early-stage osteoarthritis in unstable ankles. Arthroscopy. 2006;22 (10):1119-1125. doi:10.1016/j.arthro.2006.06.012

43. Ferkel RD, Zanotti RM, Komenda GA, et al. Arthroscopic treatment of chronic osteochondral lesions of the talus: long-term results. $\mathrm{Am}$ J Sports Med. 2008;36(9):1750-1762. doi:10.1177/ 0363546508316773

44. van Bergen CJ, Kox LS, Maas M, Sierevelt IN, Kerkhoffs GM, van Dijk CN. Arthroscopic treatment of osteochondral defects of the talus: outcomes at eight to twenty years of followup. J Bone Joint Surg Am. 2013;95(6):519-525. doi:10.2106/JBJS.L.00675

45. Vega J, Golanó P, Peña F. Iatrogenic articular cartilage injuries during ankle arthroscopy. Knee Surg Sports Traumatol Arthrosc. 2016;24:1304-1310.

46. Sammarco VJ. Complications of lateral ankle ligament reconstruction. Clin Orthop Relat Res. 2001;391:123-132. doi:10.1097/00003086-200110000-00013

\section{Publish your work in this journal}

Orthopedic Research and Reviews is an international, peer-reviewed, open access journal that focusing on the patho-physiology of the musculoskeletal system, trauma, surgery and other corrective interventions to restore mobility and function. Advances in new technologies, materials, techniques and pharmacological agents are particularly welcome. The manuscript management system is completely online and includes a very quick and fair peer-review system, which is all easy to use. Visit http://www.dovepress.com/testimonials.php to read real quotes from published authors. 